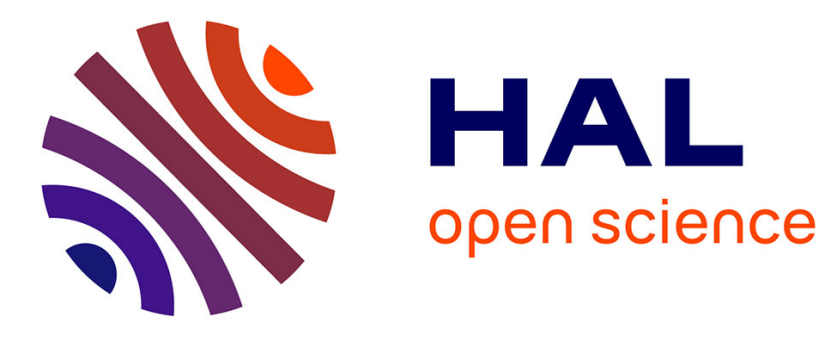

\title{
Application des dispositifs MISS aux inverseurs à haute capacité de charge
}

Gérard Sarrabayrouse, A. Essaid, J. Buxo

\section{To cite this version:}

Gérard Sarrabayrouse, A. Essaid, J. Buxo. Application des dispositifs MISS aux inverseurs à haute capacité de charge. Revue de Physique Appliquée, 1982, 17 (10), pp.681-685. 10.1051/rphysap:019820017010068100 . jpa-00245045

\section{HAL Id: jpa-00245045 https://hal.science/jpa-00245045}

Submitted on 1 Jan 1982

HAL is a multi-disciplinary open access archive for the deposit and dissemination of scientific research documents, whether they are published or not. The documents may come from teaching and research institutions in France or abroad, or from public or private research centers.
L'archive ouverte pluridisciplinaire HAL, est destinée au dépôt et à la diffusion de documents scientifiques de niveau recherche, publiés ou non, émanant des établissements d'enseignement et de recherche français ou étrangers, des laboratoires publics ou privés. 


\title{
Application des dispositifs MISS aux inverseurs à haute capacité de charge
}

\author{
G. Sarrabayrouse, A. Essaid et J. Buxo \\ Laboratoire d'Automatique et d'Analyse des Systèmes (L.A.A.S.-C.N.R.S.), \\ 7, Avenue du Colonel Roche, 31400 Toulouse, France
}

(Reçu le 7 mai 1982, accepté le 12 juillet 1982)

\begin{abstract}
Résumé. - Il est montré que les propriétés des dispositifs MISS peuvent être exploitées pour la réalisation d'inverseurs rapides pouvant conduire de très fortes charges capacitives.

Abstract. - It is shown that the MISS can be used efficiently as a very low-consuming space buffering stage which permits to drive large capacitive loads and is therefore appropriate for integrated circuits.
\end{abstract}

1. Introduction. - La caractéristique statique courant-tension d'un dispositif Métal-Isolant TunnelSilicium (n, p)-Silicium $\left(\mathrm{p}^{+}, \mathrm{n}^{+}\right)$(MISS) polarisé à travers une résistance de charge $R_{\mathrm{L}}$ présente deux zones stables d'impédances positives très différentes, séparées par une zone instable d'impédance négative (voir Fig. 1) [1-3]. La résistance dans l'état bloqué est de l'ordre de plusieurs centaines de kiloohms à quelques méghoms alors que la résistance dans l'état passant est de l'ordre de la centaine d'ohms. La commutation

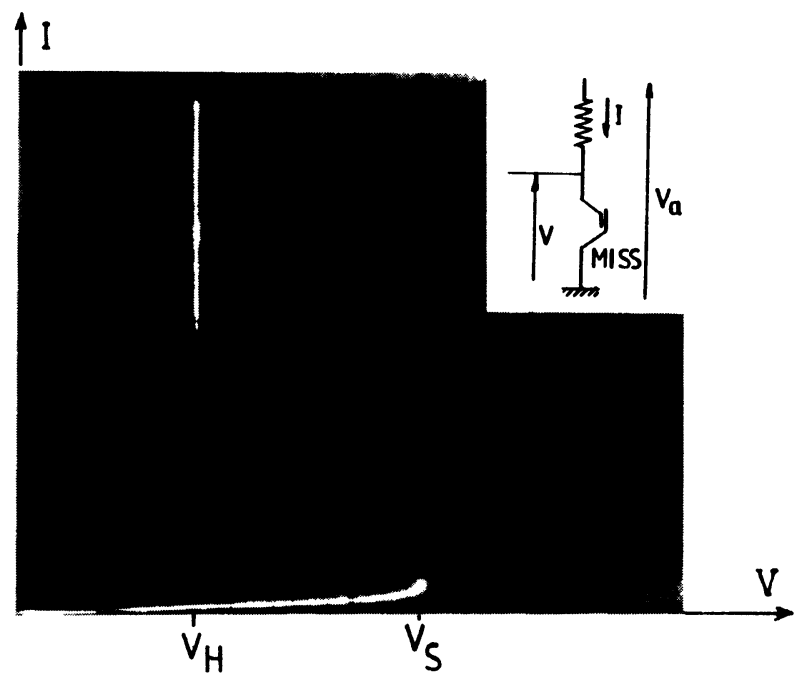

Fig. 1. - Caractéristique courant-tension d'un dispositif MISS. Mode de polarisation : $V: 0,5 \mathrm{~V} /$ div. ; $I: 10 \mu \mathrm{A} /$ div.

$[I-V$ curve of a MISS device. Biasing conditions : $V$ : $0.5 \mathrm{~V} / \mathrm{cm} ; I: 10 \mu \mathrm{A} / \mathrm{cm}$.] entre les deux états stables intervient lorsque la tension aux bornes du dispositif $V$ excède une valeur seuil $V_{s}$.

Les applications du dispositif précédemment envisagées mettent à profit l'existence des deux zones stables, afin de définir deux états de polarisation du dispositif correspondant à une même tension $V_{\mathrm{a}}$ appliquée à l'ensemble MISS-résistance de charge. Ainsi ont été étudiés un point mémoire $[4,5]$, un registre à décalage $[6,7]$, un détecteur optique [8].

L'application envisagée dans cet article utilise non plus l'existence de deux états de polarisation, mais son aptitude à conduire un courant élevé pendant un laps de temps très court lorsqu'il est polarisé à travers une capacité sous une tension continue.

Cette propriété permet, en associant un ou deux dispositifs MISS et un transistor MOS, de définir un inverseur permettant de commander un étage de très forte capacité (supérieure à $100 \mathrm{pF}$ ) d'entrée en un temps très bref (inférieur à $50 \mathrm{~ns}$ ) sans consommation notable en dehors des périodes de commutation.

\section{Le dispositif MISS polarisé à travers une capacité} de charge. - Considérons le circuit représenté sur la figure 2. Lorsque un échelon de tension $V_{\mathrm{a}}$ est appliqué, la caractéristique courant-tension du dispositif MISS est représentée sur la figure 3 . Les figures $3 a$ et $3 b$ sont relatives à un échelon de tension d'entrée $V_{\mathrm{a}}$ d'amplitude inférieure à la tension de seuil $V_{\mathrm{s}}$ du dispositif MISS. Sur la figure $3 a$ sont représentées les caractéristiques $V(t)$ et $I(t)$. Le niveau haut est fixé par le rapport de la capacité $C(82 \mathrm{pF}$ dans le cas présent $)$ à celle du dispositif MISS. En effet, celui-ci restant dans l'état forte impédance intervient dans la fenêtre temporelle analysée surtout par sa capacité. 


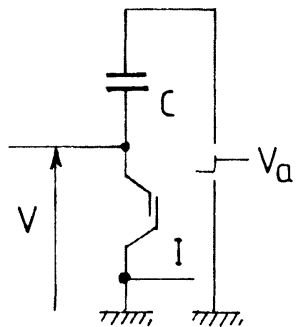

Fig. 2. - Polarisation d'un dispositif MISS à travers une capacité.

[MISS device biased through a load capacitor.]

La caractéristique $I(V)$ parcourue durant la fenêtre temporelle est représentée sur la figure $3 b$ (la portion $\mathrm{BC}$ est relative à l'oscillation parasite visible sur la figure $3 a$ ).

Lorsque l'échelon d'entrée est tel que la tension $V$ excède la valeur $V_{\mathrm{s}}$ (Figs. $3 c$ et $3 d$ ) au bout d'un temps de délai $t_{\mathrm{D}}$, le dispositif commute dans l'état faible impédance; le courant croît alors très rapidement

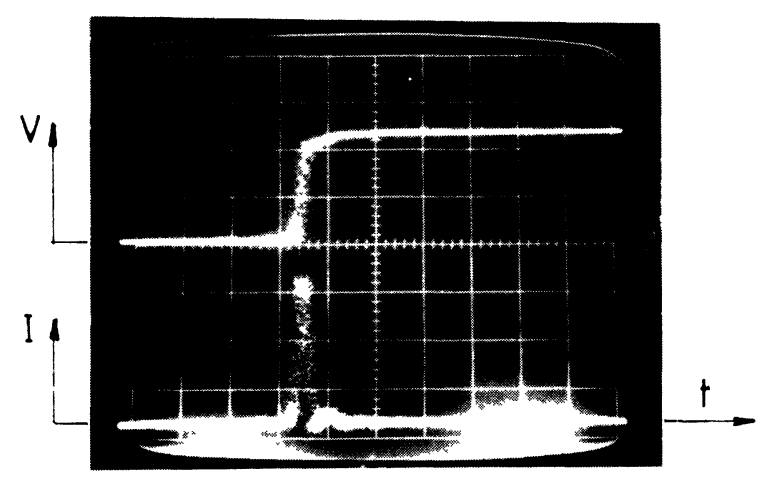

(a)

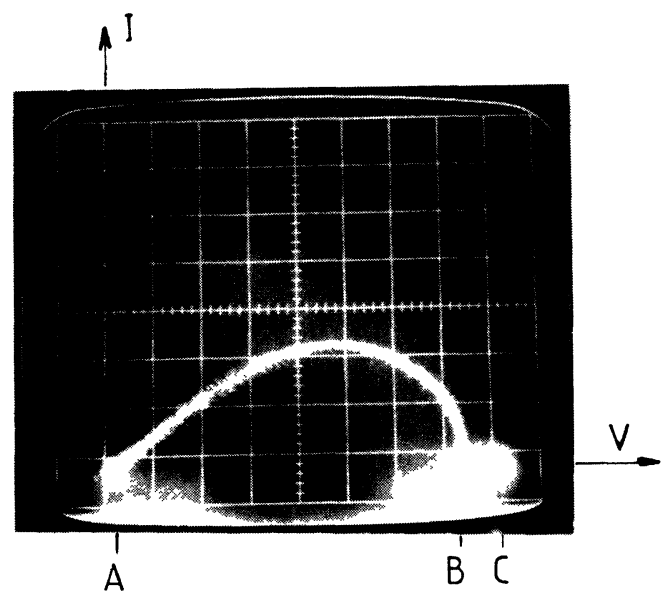

(b) durant la charge de la capacité $C$ (portion DE), puis décroît jusqu'au passage du dispositif MISS dans l'état forte impédance (portion EF). Le courant reste alors très faible (relativement à l'amplitude du pic de courant) pendant le retour de la tension $V$ à zéro (portion FG non continue dans la fenêtre temporelle analysée). La valeur de la tension $V$ immédiatement après la commutation est approximativement fixée par la tension de maintien $V_{\mathrm{H}}$ du dispositif MISS (voir Fig. 1).

3. Utilisation d'un dispositif MISS comme charge d'un transistor MOS. - Les résultats du paragraphe précédent peuvent être exploités pour réaliser un circuit inverseur pouvant effectuer la charge d'une capacité très élevée en un temps très court sans consommation en dehors de la période de commutation. En effet, considérons le circuit suivant (Fig. 4) constitué de la mise en série d'un dispositif MISS et d'un transistor MOS.

Afin d'analyser son fonctionnement en régime statique, nous faisons l'hypothèse (qui sera vérifiée lors

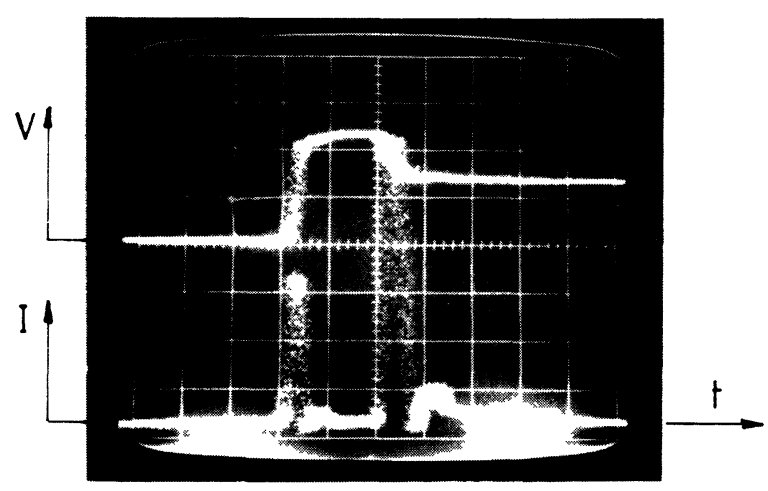

(c)

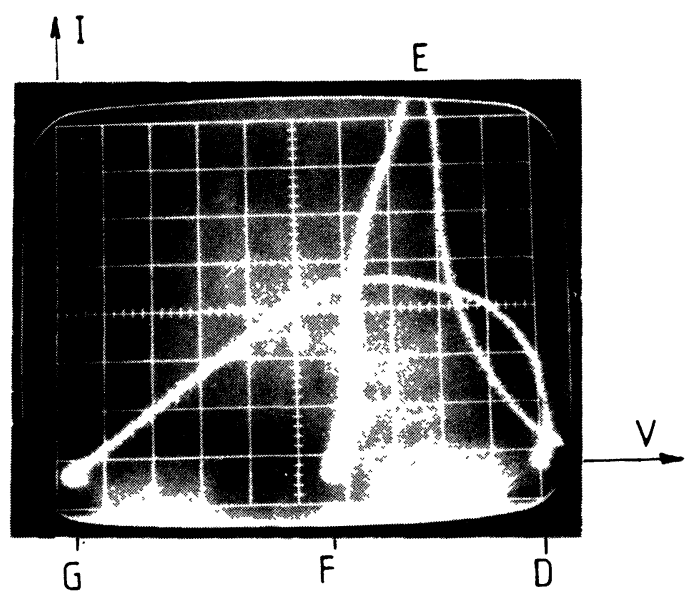

(d)

Fig. 3. - Caractéristique $I(t),(a, c)$ et $I(V),(b, d)$ d'un dispositif MISS polarisé suivant la figure $2: a)$ et $\left.b): V_{\mathrm{a}}<V_{\mathrm{s}} ; c\right)$ et d) $\left.: V_{\mathrm{a}}>V_{\mathrm{s}} . a\right)$ et $\left.c\right): V: 2 \mathrm{~V} /$ div., $I: 4 \mathrm{~mA} /$ div., $t: 20 \mathrm{~ns} /$ div. $b$ ) et $\left.d\right): V: 0,5 \mathrm{~V} /$ div., $I: 4 \mathrm{~mA} /$ div. Les courbes sont obtenues sur un oscilloscope à échantillonnage Tektronix RM567, les courants sont mesurés par une sonde Tektronix CT1.

$[I(t)(a, c)$ and $I(V)(b, d)$ curves for a MISS biased as on figure 2. The current is measured via a Tektronix CT1 probe and the curves are obtained on a Tektronix RM 567 sampling scope. a) and $b): V_{\mathrm{a}}<V_{\mathrm{s}} . c$ ) and $\left.\left.d\right): V_{\mathrm{a}}>V_{\mathrm{s}} . a\right)$ and $c$ ) :V: $2 \mathrm{~V} / \mathrm{cm}, I: 4 \mathrm{~mA} / \mathrm{cm}, t: 20 \mathrm{~ns} / \mathrm{cm} . b)$ and $d): V: 0.5 \mathrm{~V} / \mathrm{cm}, I: 4 \mathrm{~mA} / \mathrm{cm}$. 


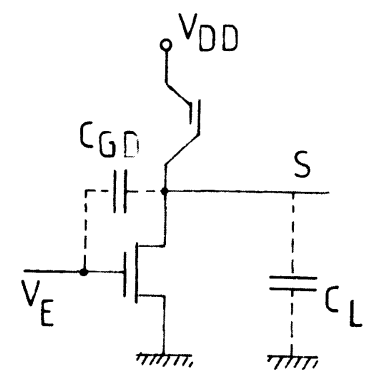

Fig. 4. - Polarisation d'un transistor MOS à travers un dispositif MISS.

[MOS transistor biased through a MISS device.]

de l'analyse du comportement dynamique) que le dispositif MISS se trouve dans l'état forte impédance. Dans ces conditions, lorsque la tension d'entrée $V_{\mathrm{E}}$ est inférieure à la tension de seuil $V_{\mathrm{T}}$ du transistor MOS, ce dernier ne conduit pas et la sortie $S$ polarisée par l'alimentation $V_{\mathrm{DD}}$ à travers la résistance $R_{\mathrm{OFF}}^{\mathrm{MISS}}$ du dispositif MISS se trouve au niveau $V_{\text {DD }}$.

Lorsque la tension $V_{\mathrm{E}}$ est supérieure à $V_{\mathrm{T}}$, le transistor MOS conduit et, si la résistance du canal du transistor est $R_{\mathrm{ON}}^{\mathrm{MOS}}$, la sortie $\mathrm{S}$ se trouve polarisée au niveau $V_{0}$ tel que :

$$
V_{0}=\frac{V_{\mathrm{DD}} R_{\mathrm{ON}}^{\mathrm{MOS}}}{R_{\mathrm{ON}}^{\mathrm{MO}}+R_{\mathrm{OFF}}^{\mathrm{MISS}}} \approx V_{\mathrm{DD}} R_{\mathrm{ON}}^{\mathrm{MOS}} / R_{\mathrm{OFF}}^{\mathrm{MISS}}
$$

Afin que dans cet état le dispositif MISS soit dans l'état forte impédance, il est nécessaire que la relation suivante :

$$
V_{\mathrm{DD}}\left(1-\frac{R_{\mathrm{ON}}^{\mathrm{MOS}}}{R_{\mathrm{OFF}}^{\mathrm{MISS}}}\right)<V_{\mathrm{s}}
$$

qui traduit le fait que la tension aux bornes du dispositif MISS est inférieure à sa tension de seuil, soit vérifiée.

En conséquence, si la condition suivante est respectée :

$$
V_{0}<V_{\mathrm{T}}<V_{\mathrm{DD}}
$$

l'ensemble se comporte comme un inverseur dont la résistance de charge $R_{\mathrm{OFF}}^{\text {MISS }}$ est élevée.

Du point de vue dynamique, supposons que l'entrée E soit au niveau de polarisation bas $V_{0}$ et passe au niveau haut $V_{\mathrm{DD}}$, le front de montée est transmis par l'intermédiaire de la capacité grille-drain $C_{\mathrm{GD}}$ (voir Fig. 4) à la sortie. Cependant, ceci a pour effet de maintenir le dispositif MISS dans l'état forte impédance puisque l'impulsion positive transmise tend à diminuer (ou même à inverser) la tension aux bornes du dispositif MISS. Donc au cours de cette transition, le dispositif MISS n'intervient que comme une résistance élevée et la décharge de s'effectue à travers le transistor MOS.

Lorsque l'entrée passe du niveau haut $V_{\mathrm{DD}}$ au niveau bas $V_{0}$, le front de descente est transmis par $C_{\mathrm{GD}}$ sous la forme d'une impulsion négative à la sortie. Ceci a pour effet d'accroître la tension aux bornes du dispositif MISS qui, la sortie étant au niveau $V_{0}$, était polarisée près de sa tension de seuil avant le changement d'état. Si la capacité $C_{\mathrm{GD}}$ a une valeur suffisante pour que l'impulsion transmise à la sortie soit d'une amplitude supérieure à $V_{\mathrm{s}}+V_{0}-V_{\mathrm{DD}}$ pendant une durée supérieure à $t_{\mathrm{D}}$, le dispositif commute dans l'état faible impédance, assurant ainsi la charge de la capacité de sortie en un temps très bref puisque le courant pendant cette phase peut être élevé. Dès que la sortie atteint un niveau de polarisation égal à $V_{\mathrm{DD}^{-}}-V_{\mathrm{H}}$, le dispositif retourne à l'état forte impédance comme dans le paragraphe précédent et le courant prend une valeur très faible durant le passage de la sortie de $V_{\mathrm{DD}}-V_{\mathrm{H}}$ à $V_{\mathrm{DD}}$.

En résumé, l'introduction d'un dispositif MISS comme charge d'un transistor MOS a pour effet de permettre la charge très rapide d'une capacité de sortie élevée du fait de la faible résistance du dispositif pendant cette phase, tout en bénéficiant d'une résistance de charge élevée en dehors des périodes de transition, et particulièrement lorsque la sortie se trouve à l'état bas et que le transistor MOS conduit.

4. Utilisation de deux dispositifs MISS. - Dans le paragraphe précédent, le dispositif MISS n'agit que lors de la charge de la capacité de sortie. Il est possible d'obtenir le même résultat en ce qui concerne la décharge en ajoutant un autre dispositif en parallèle entre le drain et la source du transistor MOS. La décharge s'effectue alors à travers le nouveau dispositif MISS dont le mode de fonctionnement est analogue à celui décrit dans le paragraphe précédent.

Dans ces conditions, l'inverseur est représenté sur la figure 5 et les caractéristiques électriques des divers éléments doivent être telles que les relations suivantes soient satisfaites :

$$
\begin{gathered}
X=\frac{R_{\mathrm{ON}}^{\mathrm{MOS}} \ll R_{\mathrm{OFF}}^{\mathrm{MISS} 2}<R_{\mathrm{OFF}}^{\mathrm{MISS} 1}}{\frac{R_{\mathrm{OFF}}^{\mathrm{MISS} 2}}{R_{\mathrm{OFF}}^{\mathrm{MISS}}+R_{\mathrm{OFF}}^{\mathrm{MISS} 1}}<V_{\mathrm{S} 2} / V_{\mathrm{DD}}} \\
Y=\frac{R_{\mathrm{ON}}^{\mathrm{MOS}}}{R_{\mathrm{ON}}^{\mathrm{MOS}}+R_{\mathrm{OFF}}^{\mathrm{MISS} 2}}<V_{\mathrm{S} 1} / V_{\mathrm{DD}} \\
V_{\mathrm{DD}}<V_{\mathrm{S} 1}+V_{\mathrm{S} 2} \\
Y<V_{\mathrm{T}} / V_{\mathrm{DD}}<1-X .
\end{gathered}
$$

La relation (4) doit être satisfaite afin que l'excursion logique entre niveau haut et niveau bas soit convenable.

Les relations (5), (6) et (7) dans lesquelles $V_{\mathrm{S} 1}$ et $V_{\mathrm{S} 2}$ sont les tensions de seuil des dispositifs MISS 1 et MISS 2, traduisent le fait que les deux dispositifs MISS sont dans l'état forte impédance en régime statique. Enfin, la relation (8) traduit le fait qu'un étage inverseur peut conduire un étage suivant identique. 


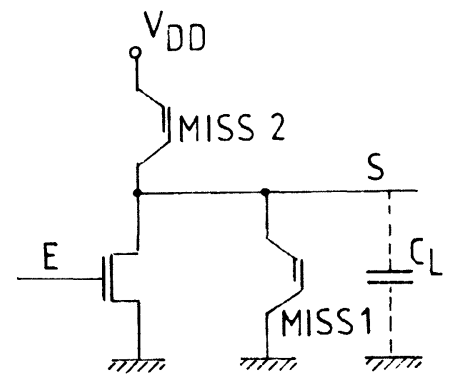

Fig. 5. - Inverseur utilisant deux dispositifs MISS.

[Inverter using two MISS devices.]

5. Résultats expérimentaux. - L'inverseur réalisé est semblable à celui de la figure 5 . Cependant, une résistance de $30 \mathrm{k} \Omega$ a été ajoutée entre le transistor MOS et la masse pour mettre en évidence l'influence du dispositif MISS 1. En effet, la capacité de sortie $C_{\mathrm{L}}$ est de $130 \mathrm{pF}$, de sorte que le temps de décharge à travers le transistor MOS est supérieur à $4 \mu \mathrm{s}$.

Le transistor MOS utilisé est un 2 N6660 et les dispositifs MISS ont été obtenus suivant un processus technologique déjà décrit dans la littérature [2]. Les tensions de seuil sont respectivement $V_{\mathrm{s} 1}=2,1 \mathrm{~V}$, $V_{\mathrm{S} 2}=1,9 \mathrm{~V}$ et la tension d'alimentation $V_{\mathrm{DD}}=3 \mathrm{~V}$.

Les résultats obtenus sont représentés sur les figures $6 a$ et $6 b$ en ce qui concerne respectivement la charge et la décharge de la capacité de sortie. Il apparaît que dans les deux cas, la transition s'effectue en moins de 50 ns. Par ailleurs, il apparaît sur la figure $6 b$ que le courant en régime permanent est inférieur à $1 \mu \mathrm{A}$, valeur très faible comparée à la valeur du courant lors de la charge de la capacité $C_{\mathbf{L}}$ $(I \approx 7 \mathrm{~mA})$.

6. Conclusion. - Les résultats que nous venons de présenter montrent que les dispositifs MISS permettent de définir, en association avec un transistor MOS, un inverseur capable de conduire des charges capacitives élevées $(100 \mathrm{pF})$ en des temps très brefs $(50 \mathrm{~ns})$ avec un rapport de courant de charge au courant de repos de l'ordre de $10^{4}$. Par ailleurs et bien que les résultats présentés ici aient été obtenus à l'aide d'un circuit hybride, la surface du même circuit sous forme intégrée peut être estimée de l'ordre de 6000 à $7000 \mu \mathrm{m}^{2}$. Il est à remarquer qu'un inverseur utilisé en " superbuffer ", réalisé en technologie canal $\mathrm{N}$, transistor de charge implanté et présentant les mêmes performances, occuperait une surface de plus de $40000 \mu \mathrm{m}^{2}$ et le courant de repos serait de plusieurs milliampères.

Par ailleurs à des fins de comparaison avec une technologie C.MOS, les inverseurs CD 4007 dévelop-

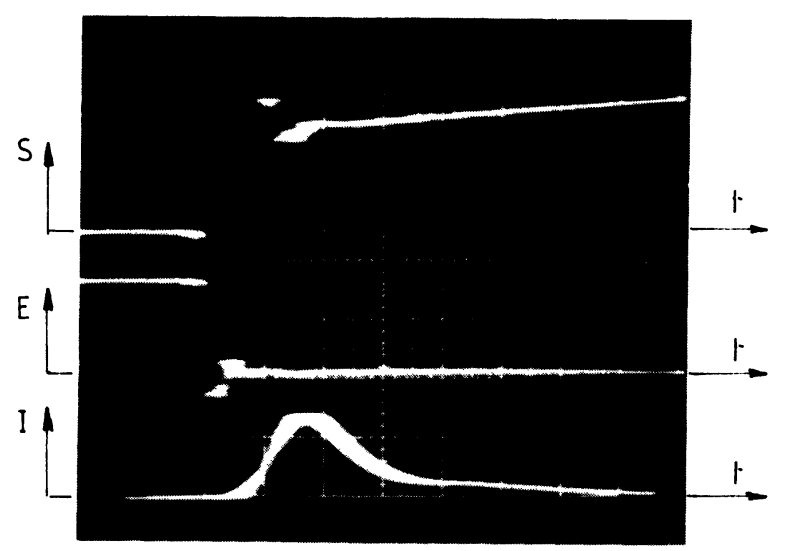

(a)

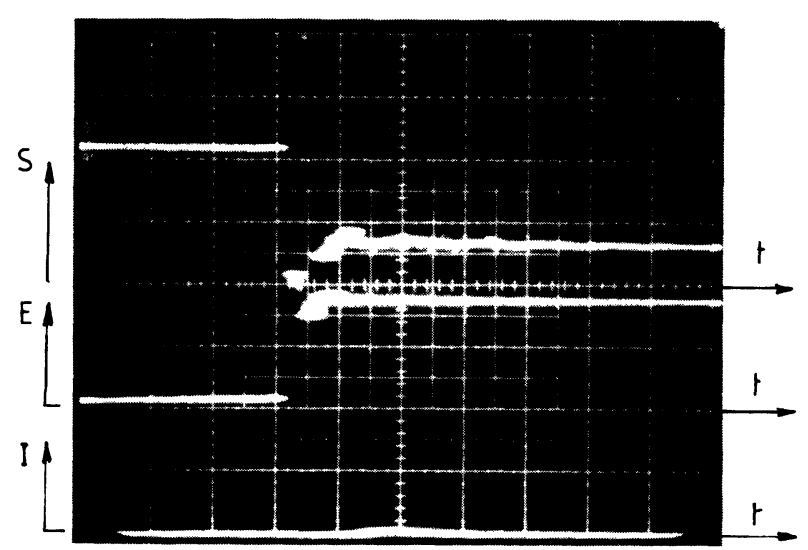

( b )

Fig. 6. - Caractéristique entrée-sortie de l'inverseur réalisé : a) charge de la capacité de sortie : $\mathrm{E}: 2 \mathrm{~V} /$ div., $\mathrm{S}: 1 \mathrm{~V} /$ div., $I: 5 \mathrm{~mA} /$ div., $t: 50 \mathrm{~ns} /$ div. b) décharge de la capacité de sortie : $\mathrm{E}: 2 \mathrm{~V} /$ div., $\mathrm{S}: 1 \mathrm{~V} /$ div., $I: 10 \mathrm{~mA} /$ div., $t: 50 \mathrm{~ns} /$ div. L'oscilloscope utilisé est un Tektronix 7623A/7A18 et le courant est mesuré via une sonde Tektronix 134 (qui introduit un retard de l'ordre de $50 \mathrm{~ns}$ ).

[Input-output curves of the inverter : a) charging the output : $\mathrm{E}: 2 \mathrm{~V} / \mathrm{cm} . b$ ) discharging the output $: \mathrm{E}: 2 \mathrm{~V} / \mathrm{cm}$, $I: 10 \mu \mathrm{A} / \mathrm{cm}, t: 50 \mathrm{~ns} / \mathrm{cm}$. The current is measured via a Tektronix 134 probe (giving rise to a $50 \mathrm{~ns}$ delay). The curves are obtained on a Tektronix 7623A/7A18 scope.]

pés par RCA permettent de commuter $100 \mathrm{pF}$ en 120 ns [9].

Remerciements. - Nous remercions le personnel de la Centrale de Technologie du Laboratoire pour la réalisation des échantillons. 


\section{Bibliographie}

[1] Kroger, H., Wegener, H. A. R., Appl. Phys. Lett. 23 no 7 (1973) 397.

[2] Sarrabayrouse, G., Buxo, J., Sebaa, J. P., Essaid, A. IEE Proc. 128 Pt I no 2 (1981) 53.

[3] Millan, J., Serra-Mestre, F., Buxo, J., Revue Phys. Appl. 14 no 11 (1979) 921.

[4] Simmons, J. G., El BADRY, A., The Radio and Electronic Engineer 48 n $^{\circ} 5$ (1978) 215.

[5] Buxo, J., Amat, L., Sarrabayrouse, G., 4th European Conference on Electrotechnics Stuttgart, March 2428, 1980.
[6] Yамамото, T., Morimoto, M., Solid State Electron. 19 (1976) 701.

[7] Fiore de mattos, A. C., Sarrabayrouse, G., Buxo, J., ESSAID, A., 11th European Solid State Device Research Conference, Toulouse, September 14-17, 1981.

[8] Nassibian, A. G., Calligaro, R. B., Simmons, J. G., Solid State Electron. Device 2 no 5 (1978) 149.

[9] Données issues de RCA Solid State DATA BOOK Series SSD 250. 